\title{
Anabases
}

ANABASES Traditions et réceptions de l'Antiquité

19 | 2014

Varia

\section{Le prix du papyrus dans l'Antiquité grecque}

Annales d'histoire économique et sociale, $1^{\text {re }}$ année, $n^{\circ} 1$, 1929, p. 3-12

\section{Gustave Glotz}

\section{(2) OpenEdition}

\section{Journals}

Édition électronique

URL : http://journals.openedition.org/anabases/4765

DOI : 10.4000/anabases.4765

ISSN : 2256-9421

Éditeur

E.R.A.S.M.E.

\section{Édition imprimée}

Date de publication : 1 avril 2014

Pagination : 301-310

ISSN : $1774-4296$

Référence électronique

Gustave Glotz, «Le prix du papyrus dans I'Antiquité grecque », Anabases [En ligne], 19 | 2014, mis en ligne le 01 avril 2017, consulté le 21 octobre 2019. URL : http://journals.openedition.org/anabases/ 4765 ; DOI : 10.4000/anabases.4765 


\section{LE PRIX DU PAPYRUS DANS L'ANTIQUITE GRECQUE ${ }^{1}$}

Les érudits se sont souvent demandé quel était dans les pays de la Grèce ancienne le prix du papier en fibres de papyrus ${ }^{2}$. La question n'est pas seulement d'un grand intérêt pour l'histoire de la civilisation; comme il s'agit d'une fabrication et d'un commerce exclusivement égyptiens, elle a aussi son importance dans l'histoire économique et, comme on verra, dans l'histoire des relations internationales.

Jusqu'en 1912, on ne trouvait sur cette question, dans nos documents tant littéraires qu'épigraphiques, que trois indications, d'ailleurs contradictoires. Tout en regrettant la rareté des renseignements, la plupart des auteurs soutenaient que le papyrus a été cher de tout temps. Seul, Gardthausen était d'avis qu'il a été cher avant et après la période hellénistique, mais que pendant quatre siècles le bon marché de la matière première et de la main-d'cuvre, ainsi que les facilités de la fabrication, permirent à l'Égypte ptolémasque de fournir au monde méditerranéen un produit bon marché. Lorsqu'en 1912 Durrbach eut publié les comptes des hiéropes dẻliens de 314 à 250 , j'ai fait observer, dans un article Sur le prix des denrées à Délos ${ }^{3}$, que ces comptes fournissaient un bon nombre d'indications nouvelles sur le prix du papyrus et donnaient un démenti à tous les auteurs qui s'étaient occupés de la question. Mais je suis obligé de constater que cet article est resté dans la pénombre où sont souvent plongés les écrits confiés aux revues scientifiques: Schubart, dans son excellente Einführung in die Papyruskunde, parue en 1918, dit encore (p. 39) : "Ueber die Preise des Papyrus wissen wir trotz vereinzelten Angaben ungefähr nichts. Billig war er nicht"; et il donne les raisons de la cherté qu'il admet pour toute l'antiquité, sans distinction de temps. Maintenant que Durrbach a publié une seconde série d'inseriptions

1. Communication faite au Congrès international des Sciences historiques a Oslo (aobt 1928).

2. Volr Ex. Egarn, Hist. de la critique chez les Grecs, 1849, p. 85 et ss.; La littér. grecque, p. 29 et ss.; Sur le prix du papier dans $l^{\prime}$ antiquité (Mem, d'hist. anc. et de philol., 1863, D. 135-

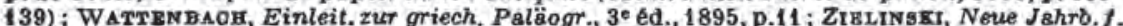
klass. Alt., t. I X, 1906, p. 269 ; Tн. BrRT, Die Buchrolle in der Kunst, 1907, col. 7-8, 26-29; Dzistzko, art. Archive dans la Realencycl. de Pauly-Wissowa, t. II, col. 553 et ss.; art. Buch et Buchhandel, ib., t. III, col. 975, 984 et ss.; Untersuchungen iber ausgewählle Kapilel des sntiken Buchwesens, p. 39-42 ; W ONgor, art. Charta dans Pauly-Wissowa, t. III, col. 2191 ; Wrin. SczoBART, Das Buch bei den Griechen und Römern, $1^{\text {ro }}$ éd., 1907, p. 27 et $8 s . ; 2^{\circ}$ éd., D. 34 8s. ; LApays, art. Liber et Papyrus, dans le Dict. des Antiq.; V. Gardtandzsn, Griech. Palaeogrsphie, $2^{\circ}$ ed., t. I, Das Buch im Alt. und im byzant. Mittelalter, 1911, D. 65-69. $65-66$.

3. Journ. des Sav., 1913, p. 28-29 ; cf. Anumx, Hist, du texte de Platon, 1915, p. 1-2, 
déliennes (1926) et va en publier uné troisième dont j'ai eu les épreuves entre les mains, et qu'ainsi je dispose de données nouvelles pour les années 231-179, je voudrais reprendre la question dans l'ensemble. Il en vaut la peine, puisqu'aux trois indications de jadis, qui s'appliquent aux années 407,333 et 322 , s'en ajoutent indépendamment de celles que fournit la papyrologie - dix-huit autres, qui se répartissent sur treize années comprises entre 296 et 179.

Nous savons que le papyrus égyptien était importé en Grèce depuis le $\mathrm{vI}^{\mathrm{e}}$ siècle; mais il y était rare à cause du prix. C'est pour cela, nous dit Hérodote, que Ies Ioniens ont longtemps employé, pour écrire, des peaux de brebis et de chèvres (âtộf́paı), ce que font encore, ajoutet-il, les barbares, évidemment ceux de l'Asie ${ }^{1}$. A la fin du ve siècle, les précieuses feuilles étaient bien plus répandues en Grèce, mais restaient chères. En 407, les épistates préposés aux travaux de l'Érechtheion en achètent deux pour y transcrire les copies de leurs comptes qui doivent être déposés aux archives; ils les payent 1 drachme 2 oboles la pièce ${ }^{2}$. C'est un prix élevé en un temps où la journée de travail vaut une drachme, même pour un architecte ${ }^{3}$.

Il est vrai qu'on oppose à ce prix, consigné dans un acte officiel, celui qu'on croit pouvoir déduire d'un texte littéraire. Platon fait dire a Socrate qu'on peut trouver sur le carreau de l'agora le Traité sur la nature d'Anaxagore pour une drachme tout au plus". Dziatzko a soutenu que, si le manuscrit valait une drachme, le papier n'en valait certainement pas plus du tiers et que, le volume se composant de plusieurs feuillets, le prix du feuillet n'atteignait pas une obole ${ }^{5}$. Mais les exemplaires dont Socrate parle avec le sourire sont des livres de rebut. Les bouquinistes du marché n'avaient pas le moins du monde la prétention de tirer de bons "rossignols" le prix du papier neuf ni, à plus forte raison, le prix du papier augmenté du salaire payé jadis au scribe, salaire qui, à lui seul, représentait plus d'une journée de travail. Platon nous donne done un renseignement précieux sur la vente des livres d'occasion, il ne dit rien sur la valeur du papyrus dans la Grèce de son temps.

Le prix fort de l'an 407 se maintient encore pendant trois quarts de siècle. En effet, à Épidaure, d'après les comptes de la Thymélè,

1. Hêr., V, 58,3 ; cf. Eurip., tr. 629 ; Diod., II, 32.

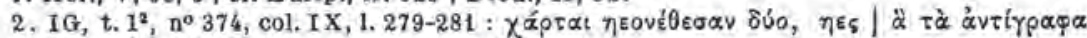

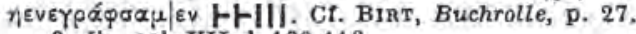

3. Ib., col. VII, 1.109-110.

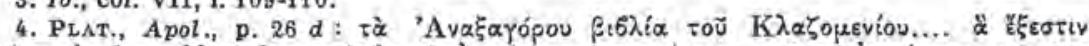

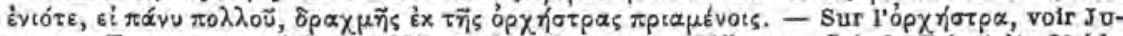
DEICH, Topogr. von Ath., p. 305, n. 13 ; IWAN vON MÜLLER, Griech. Privatalt., $2^{\circ}$ éd., p. 253.

5. Dzintzko, Untersuch, p. 40-41; Cf. Wilamowitz, Hermes, t. XXI, 1886, p. 603, note; GARDTHAUars, op. cit., p. 67-68. 
on donne 41 /2 oboles éginétiques, c'est-à-dire une drachme et une demi-obole attiques, pour une feuille sur laquelle doit être consigné un cahier de charges ${ }^{1}$. On voudrait avoir ici une date certaine. Malheureusement, tout ce qu'on croit savoir, c'est que l'achat en question a été fait dans la seizième au moins et, peut-être, dans la vingt-sixième année depuis le commencement des travaux en cours, et que ces travaux ont commencé vers 360 : donc vers $344-334^{2}$. Voilà qui est bien vague. Je crois pourtant qu'on peut préciser. Quatre ans avant cet achat de papyrus, les comptes mentionnent un achat de plomb à un prix double du prix ordinaire ${ }^{3}$. Une telle hausse ne peut s'expliquer que par une cause pareille à celle qui a produit le même effet dans les dernières années du ve siècle, c'est-à-dire par un arrêt total de l'exploitation minière dans le bassin du Laurion 4. L'une de ces crises a été déterminée par la présence des Spartiates à Décélie et la désertion des esclaves; l'autre n'a pu l'être que par l'arrivée de l'armée macédonienne sur la frontière de l'Attique, après la bataille de Chéronée. C'est donc quatre ans après $438 / 7$, en $434 / 3$, que la feuille de papier valait à Épidaure plus d'une drachme attique. Ainsi, pendant trois quarts de siècle, le prix n'a presque pas varié, au moins en temps normal.

Mais tout à coup, une dizaine d'années après, en 322, le plaidoyer contre Dionysodôros nous apprend que les grands négociants d'Athènes rédigent leurs contrats les plus importants sur des tablettes à deux chalques et sur des bouts de papier tout aussi bon marehé ${ }^{5}$. Est-ce là une de ces exagérations dont les avocats sont coutumiers ? Il est bien possible, à vrai dire, que le Pseudo-Démosthène donne un prix réel pour la tablette et un prix seulement approximatif pour le papyrus. Mais il n'aurait pas osé parler comme il l'a fait, si le papyrus avait encore coûté trente-deux fois plus, comme en 407 , ou même vingt-six fois plus, comme en 333. Pourtant, on pourrait toujours ergoter là-dessus, si nous n'avions pas d'autre indication dans le même sens.

Le grand, l'inestimable avantage que présentent lés inscriptions de Délos dans l'histoire économique de l'antiquité, c'est qu'elles nous donnent des séries de prix qui s'échelonnent sur un siècle et demi. Les différences sont caractéristiques, les hausses ou les baisses ont toujours une signification qu'il importe de démèler. J'ai pu montrer il y a quelques années par un exemple typiqué ${ }^{6}$, celui d'une denrée

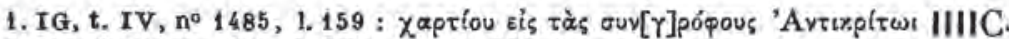

2. Volr Postow, Klio, t. XII, 1912, p. 283 et ss.

3.IG, l. c., l. 131-132. Ct. 1. 62-63, 109-110 (2 dr, 1 ob. ou 1 ob. 1/2).

4. IG, t. I', col. IX, 1. 286-289 (5 dr.). Cf. Dittenberger, Sylloge, 2o éd., no 587 , 1. 176-177; Ps. ARIsT., Econom., p. 1353 a, 15 .

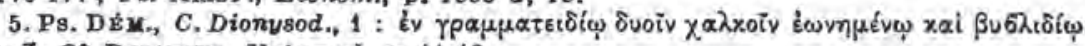

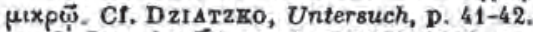

6. Rev, des Et. gr., t. XXIX, 1916, p. 281-325. 
aussi infime que la poix, comment la mercuriale délienne réflète l'histoire de la Grèce pendant un siècle. On va voir que les variations de prix subies par le papyrus ne sont pas non plus sans importance.

Les premieres indications que nous fournissent sur le prix du papier les hiéropes de Délos datent de l'an 296. Elles sont pour cette année au nombre de deux, et toutes deux conformes à celle que nous donnait l'orateur de 322. 10 Une feuille ( $\left.\chi \alpha \dot{\alpha} \tau \tau \eta_{5}\right)$ est payée à un prix qu'une mutilation de la pierre rend incertain, mais qui est peut-être d'une obole et en tout cas demeure au-dessous d'une drachme ${ }^{1}$. 2o Pour une drachme, on a plusieurs rouleaux $\left(\beta, 5 \lambda_{i \alpha}\right)^{2}$, e'est- $\alpha$ dire au moins deux rouleaux d'au moins deux feuillets et probablement de plus de deux feuillets : le feuillet vaut donc 1 obole $1 / 2$ au maximum; mais il est bien plus vraisemblablement d'un prix inferieur et peut même être, comme en 322 , d'un simple tétartèmorion.

Voilà done une période de vingt-six ans au moins (322-296) pour laquelle un prix bas est certifié.

Mais cette période est exceptionnelle. A partir de l'an 279 et jusqu'en 179, les comptes de Délos nous donnent seize prix pour douze années. Tous ces prix sont supérieurs, non pas seulement et de beaucoup à ceux de la période précédente, mais même à ceux de 407 et 333 .

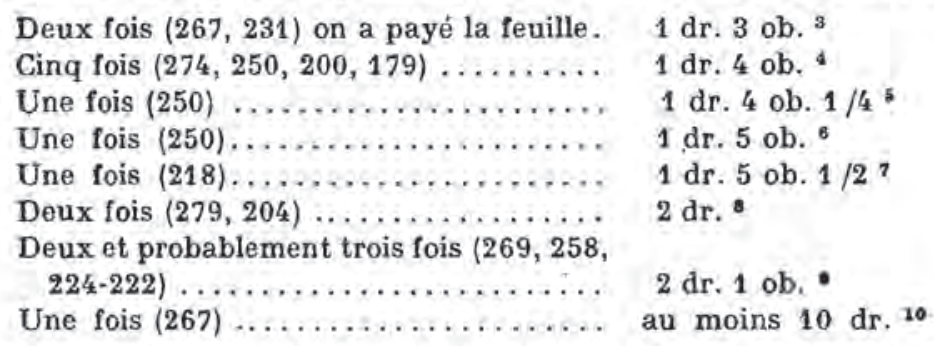

On a ainsi une série de prix qui comporte sept degrés, de 9 à

1. IG, t. XI, Ir, no 154, A, 1. $24: \chi$ áotns | ...

2. Ib., 1, $34:[\beta], 6 \lambda i \alpha .1$.

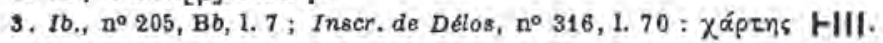

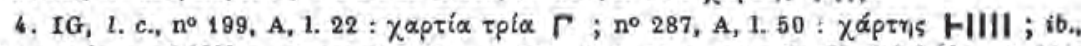

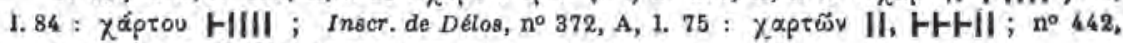

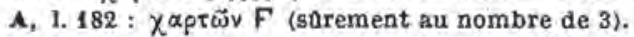

5. IG, l. c., $\mathrm{n}^{\circ} 287, \mathrm{C}, 1.1 \div \chi \alpha \dot{\rho} \tau \tau(\varsigma) ~ H I I I I T$.

6. $I b .$, A, 1. $52: \chi \alpha$ áptทs Flllll.

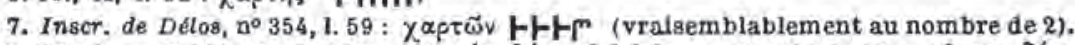

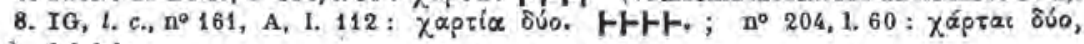
rutin HrF.

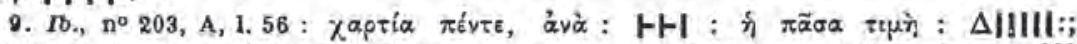

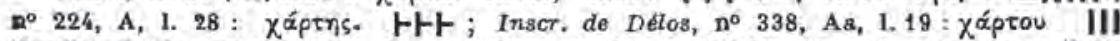
(M. Durrbach a bien voulu, a ma prlere, verifler ce chiffre sur son estampage; il reconnatt aujourd'hul au moins aussi blen des $\vdash$ que des $\mid$ : sles lectures $\vdash \vdash \vdash$ ou $\vdash$ II ne sont autorisées par aucun exemple; la restitution $F+\mid$ est donc la plus probable).

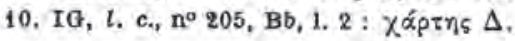


13 oboles ; après quoi, par un bond énorme, on arrive au prix unique de 10 drachmes. Il ne faut point croire, d'ailleurs, qu'au cours de ce siècle le prix ait varié selon les temps : il est de 1 drachme 4 oboles aussi bien en 274 qu'en 179 , il va dans la même année 250 de 1 drachme 4 oboles à 1 drachme 4 oboles $1 / 4$ et 1 drachme 5 oboles; bien mieux, dans la même année 267, on trouve le plus bas et le plus élevé de tous. Ce dernier prix doit, au reste, être mis à part : il n'a pu être demandé 10 drachmes ou davantage que pour la qualité de papyrus qu'une renounmée séculaire plaçait au-dessus de toutes les autres, celle qui avait une largeur de 11 doigts $(0 \mathrm{~m}, 20)$ et se distinguait par la finesse, la solidité, la blancheur et le poli ${ }^{1}$, celle qui était réservée en

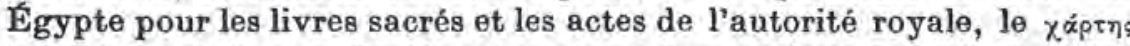

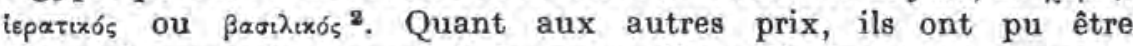
demandés pour le papyrus de bonne qualité ordinaire, à la marque de "l'Amphithéâtre ", qui se fabriquait près de l'amphithéâtre d'Alexandrie et qui avait 9 doigts de large $(0 \mathrm{~m} .17)^{3}$.

Par conséquent, si le papyrus a été bon marché en Grèce depuis le dernier quart ou le dernier tiers du $\mathrm{Iv}^{\mathrm{e}}$ siècle, il est redevenu cher dans le premier quart du $\mathrm{III}^{\mathrm{e}}$, et cette fois pour toujours. Avant de nous demander comment s'explique la période de baisse constatée dans les années 322 et 296 , précisons les dates extrêmes de cette période. On a vu par les inscriptions qu'elle peut s'allonger de dix ans $a b$ initio et de seize ans $a$ fine. Mais, si rien n'empêche de la faire commencer dès 332, il ne paralt pas qu'elle ait duré jusqu'en 279. Les comptes déliens de l'an 281 auraient pu, à cet égard, nous donner un renseignement précieux : ils mentionnent un achat de papyrus;

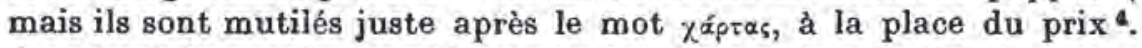
Au cas où ils auraient porté un prix bas, ils auraient daté, à deux ans près, l'événement qui modifie si fortement le prix du papyrus. Mais il est plus probable qu'ils portaient déjà un prix fort. Nous savons, en effet, que quelques années auparavant le papier n'était pas en Grèce un objet de consommation courante pour les petites bourses. Bon gré mal gré, les pauvres gens faisaient comme à l'époque lointaine de l'ostracisme, ils écrivaient sur des morceaux de pots cassés. En 283 ou 282, quand Cléanthe commença de suivre les leçons du Portique, il n'avait d'autre matière à sa disposition, pour recueillir les pensées de son maitre Zénon, que des tessons de vases et des omoplates de boufs : réduit à travailler pour vivre, le papyrus était trop cher pour lui ${ }^{5}$. Cette anecdote pourrait bien réduire de trois ou

1. Telles sont, d'après Pline (XIII, 78), les qualités qu'll faut demander au papyrus,

2. Cf. LAFAy E, art. Papyrus, p. 320 .

3. Cf. ibid.

4. IG, l. c., no $159, \mathrm{~A}, \mathrm{~L} .37$.

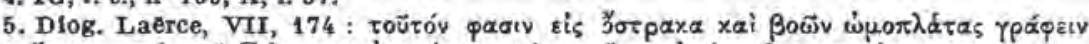

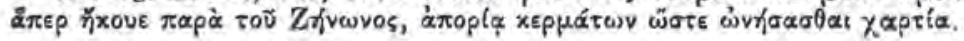


quatre ans l'intervalle dans lequel se place le retour aux prix forts. C'est du côté de l'Égypte qu'il faut nous tourner pour comprendre ce qui s'est passé une première fois dans l'intervalle de 333 à 332, une seconde fois quarante ou cinquante ans plus tard. Car la fabrication et la vente du papier étaient une industrie et un commerce exclusivement égyptiens ${ }^{1}$.

Or, tout indique qu'en Égypte le papier pouvait revenir très bon marché. La culture de la plante était fort répandue dans le Delta ; le travail demandait du soin, mais n'était pas compliqué; la maind'œuvre était abondante et ne coûtait guère que les frais d'entretien. Le prix de revient n'était donc pas élevé. C'est même cette raison qui a fait croire à Gardthausen qu'avant d'être exploité par le fisc impérial, le papyrus fut d'un prix extrêmement modéré. Mais l'hypothèse n'est juste, comme on vient de le voir, que pour un temps limité. Elle ne l'est ni pour les années antérieures à 332-322 ni pour les années postérieures à 296-282. Pourquoi ?

C'est que le monopole qui devait rendre le papyrus si cher sous les empereurs romains existait déjà sous les pharaons et fut reconstitué sous les Lagides. On voit dès lors ce qui s'est passé entre 333 et 322 et a déterminé une énorme baisse du papier. Tout simplement ceci : dans l'hiver 332-331, Alexandre a ouvert toutes grandes les portes de l'Égypte et fait affluer sur le marché grec les marchandises de l'Orient. En remplaçant les administrations nationales par la domination macédonienne, il a mis fin aux monopoles qui enrichissaient de temps immémorial le trésor des temples et la cassette royale. Alors commence pour la fabrication et la vente du papyrus un régime de liberté qui dure encore au commencement du $\mathrm{wI}^{\mathrm{e}}$ siècle. Nous savons par ailleurs et nous constatons dans les comptes de Délos que Ptolémée, fils de Lagos, satrape indépendant depuis 311 , roi depuis 305, n'avait pas encore réorganisé les monopoles en 296. Autrement, dans une année où le maître des lles, Démètrios Poliorcète, se trouvait en état de guerre avec le maitre de l'Égypte, le papyrus, plus cher déjà qu'en 322 , eut été d'un prix quasiment inabordable.

Tout semble indiquer que Ptolémée Sôter ne changea pas de politique fiscale jusqu'à son abdication en 285. Son successeur, au con-

1. Depuis que cet article a été lu au Congrès d'Oslo, j'ai trouvé par hasard, dans un texte qui m'avait complétement échappé, une eclatante confirmation des rapports a tablir entre le prix du papyrus sur le marché grec et la situation de l'Egypte. A la fin d'une lettre, adressee à Phllippe de Macédolne dans la seconde moitie de 343 , le philosophe Speusippos déclare que "le papler lui manque s pour ecrire tout ce qu'il voudrait, * tant est grande la disette de papier que le rol (Artaxerxés) a créé par la conquête de l' (Voir E. Bickermann et Jor. Sykutrus, Speusipps Brief an König Philipp, Berichte der Sschs. Akad. der Wissensch. zu Leipzig, Philol.-hist. Klasse, t. LXXX, 1928, fasc. III, p. 12,14 ; pour la date, volr p. 30 et ss.). Ce texte est d'une très grande valeur pour notre recherche. Non seulement II nous lalsse deviner que le papyrus pouvait atteindre un prix formidable dans la pérlode antérieure au dernier tiers du $1 v^{\circ}$ siecle; mals c'est le seul document qui nous dise en toutes lettres que nous avons ralson, dans la question quil nous occupe, de mettre l'hístoire économique en relations avec l'histoire politique. 
traire, revint aux traditions pharaoniques. Nous savions par les Revenue Laws que, la vingt-septième année de son règne, Ptolémée Philadelphe donna une constitution définitive à certains monopoles. C'était une réforme, non une création. Le prix payé pour le papyrus en 279 et l'anecdote de Cléanthe nous apprennent que le monopole du papier était déjả rétabli la sixième, peut-être même la deuxième année du règne. En tout cas, il fallait bien une raison de ce genre pour que le prix du papyrus fût si élevé en 279 , puisqu'à cette époque le commerce des iles avec l'Égypte était facilité par d'excellentes relations, comme le prouvent l'invitation adressée aux Nèsiotes et acceptée par eux d'assister officiellement aux fêtes olympiques d'Alexandrie et la fondation des Ptolémaia à Délos 1 .

Nous possédons assez de renseignements sur le monopole du papyrus au temps des Lagides pour qu'il soit impossible d'en révoquer l'existence en doute. La matière première était achetée aux particuliers d'après le tarif fixé par l'administration royale; le travail se faisait dans les ateliers publics, sauf le privilège réservé aux prêtres de pourvoir par leurs moyens aux besoins des temples; la vente était assurée par des détaillants qui s'approvisionnaient dans les magasins du roi ${ }^{2}$. Le prix de vente se régla donc bien moins sur le prix de revient que sur le bénéfice réclamé par le fisc.

Aussi ne pouvait-on se procurer du papier bon marché même en Egypte. On a souvent observé que, sans la nécessité de faire des économies, les sujets des Lagides n'auraient pas constamment écrit sur le verso de leurs feuilles ni surtout recouru à la misérable pratique du palimpseste. Ils auraient encore moins fait un tel usage des ostraca, s'ils avaient eu à leur disposition une matière plus commode à un prix modéré. Enfin, dans un pays où le respect des morts fut toujours poussé jusqu'au scrupule le plus délicat, les embaumeurs n'auraient pas enveloppé les momies de vieux papiers mis au rebut, s'ils avaient pu en avoir de neufs à bon compte.

Précisément, les indications que nous possédons sur le prix du papyrus en Egypte sont bien en rapport avec celles que nous donnent les inscriptions de Délos pour la même époque. On trouve, entre les unes et les autres, tantôt la différence normale qui représente le droit de sortie, les frais de transport et le bénéfice de l'intermédiaire, tantôt une différence exceptionnellement forte qu'explique une perturbation des relations commerciales causée par les événements politiques. En 251-250, tandis que les comptes des hiéropes déliens donnent deux fois le prix de 1 drachme 4 oboles, une fois celui de 1 drachme 4 oboles $1 / 4$ et une fois celui de 1 drachme 5 oboles, les comptes de Zénon men-

1. Voir Rev. des Et. gr., t. c., p. 308-309.

2. Voir Dziatzizo, Untersuch. p. 98 ; Bouchí-LeclencQ, Hist. des Lsgides, t. III, p. 267 ; WrLOKEN, Grundzilge, t. I, 1, p. 255-256. 
tionnent le prix de 1 drachme 1 obole ${ }^{1}$. La différence n'est guère plus grande au $\mathrm{nt}^{\mathrm{e}}$ siècle ; tandis qu'on paie à Délos 1 drachme 4 oboles la feuille, prix de détail, une administration égyptienne paie 100 drachmes pour 100 feuilles, 1 drachme par feuille, prix de demigros ${ }^{2}$. Il est vrai qu'à certains moments le prix s'abaisse dans le pays de production au-dessous de 1 drachme. Un compte du Fayoum

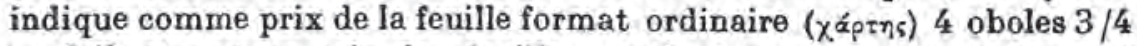

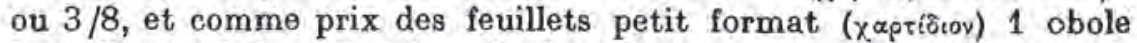
le feuillet et 8 drachmes la main de 48 ou peut-être de 50 feuillets ${ }^{3}$. Faute de date précise, nous ne pouvons, dans ce cas, faire de comparaison. Mais, vers 259-258, nous trouvons un écart énorme, Les comptes de Délos portent, en 258, le prix considérable de 2 drachmes 1 obole, peut-être bien parce que l'Égypte est impliquée dans les événements de Cyrénalque et que les risques de guerre entravent le commerce gréco-égyptien. Vers le mème temps, les comptes de Zénon mentionnent un achat de papyrus que l'éditeur croit pouvoir évaher, malgré les difficultés de lecture qu'il signale loyalement, à 40 drachmes les 60 feuilles, donc à 4 oboles la feuille 4 . Si le nombre $\xi=60$ doit vraiment être conservé pour la quantité, on peut songer, pour le prix, à remplacer $\mu$ par v, ou 40 par 50 , ce qui serait plus conforme à la mercuriale du temps. Il n'est pas impossible pourtant que le nI ${ }^{\mathrm{e}}$ siècle avant l'ère chrétienne ait déjà connu ce prix de 4 oboles qui est certifié pour le milieu du ${ }_{1}{ }^{e}$ siècle après J.-C. ${ }^{5}$. En tout cas, depuis le règne de Ptolémée Philadelphe, le tarif des papyrus en Egypte - même si, de 1 drachme 1 obole au plus haut, il descend a 4 oboles au plus bas - reste encore bien au-dessus des prix qu'on payait dans les pays importateurs comme l'Attique et Délos, à plus forte raison dans le pays producteur, avant le rétablissement du monopole.

Reste à examiner un document où Gardthausen a cru trouver une confirmation de sa théorie et qui nous fait, au contraire, mieux connastre le monopole du papier. Sur un papyrus de Tebtynis, un comogrammate mentionne, en l'an 112, la dépense suivante : xátepyov

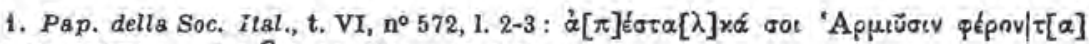
roir $[s] \chi a ́ p[\tau] \alpha \xi \varepsilon, 1-\varepsilon \models$ (5 feu llles, 5 dr. 5 ob.).

2. IV. Schubart-E. KüнN, Papyri und Ostraka der Ptolemaerzeit (Agyptische Urkunden aus den stastl. Museen zu Berlin, Griech. Urk., t. VI, 1922, p. 36, no $1233,1.3$ : $\chi \alpha(\rho \tau \alpha \varsigma) p, p)$.

3. SAyoe, dans Flin ders Petrie, Hawata, p. 34, no 245 (Cf. Preigroke, Sammelbuch oriech. Urkunden in Egypten, $n^{\circ} 5224,1.7: \chi \alpha$ áprou $f ., ; 1.38: \chi \propto \rho \tau i \delta[$ [ou, et non

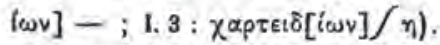

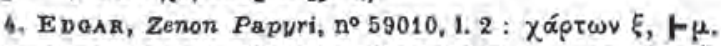

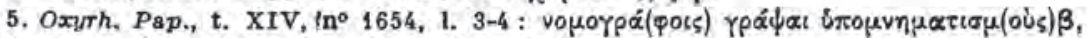

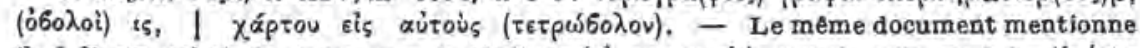
(1. 5-6) un achat de papyrus * a débiter, (sis ouvxortdv) pour le prix de $4 \mathrm{dr}$. (étépoou

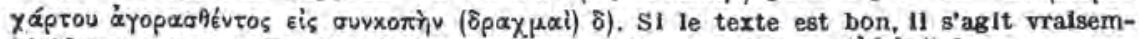

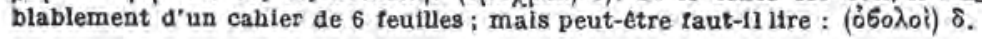




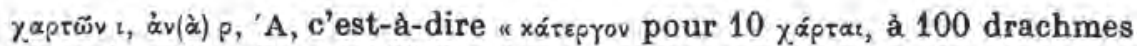
(de cuivre) la pièce, 1000 drachmes " ${ }^{1}$. Pour Crönert, qui a examiné ce texte, $\chi \alpha$ á rท̆ ne désigne pas une feuille de papyrus, mais un rouleau, une main, un scapus, de vingt feuilles ${ }^{2}$. Hypothèse purement arbitraire, Gardthausen la fait sienne pourtant et, après avoir correctement traduit xárepyov par Lohn, il continue en raisonnant comme s'il s'agissait, non d'un salaire, mais d'un prix de vente. Et voici sa conclusion : 10 mains de papyrus valent 1000 drachmes de cuivre; done une main vaut 100 drachmes; done une feuille vaut 5 drachmes, ce qui fait, à une époque où la monnaie de cuivre a fortement baissé par rapport à la monnaie d'argent, moins d'un pfennig ${ }^{3}$. Revenons tout simplement à notre texte et traduisons : "Salaire pour fabrication de 10 feuilles à 100 drachmes la pièce, 1000 drachmes ". Il s'agit d'une somme due par l'administration du monopole à un ouvrier ou à un entrepreneur de papeterie appelé plus loin yaftorotós. D'ailleurs, étant donnó que le rapport de l'argent monnayé au cuivre était alors de $1: 475$, l'ouvrier recevait 0 fr. 20 argent par feuille, et non pas $0 \mathrm{fr}$. 01, comme le voudraient Crönert et Gardthausen.

Le bénéfice du trésor royal était assez beau, puisque le prix de la feuille était pour les Egyptiens d'au moins $0 \mathrm{fr}, 65$ argent ( 4 oboles) et atteignait même $1 \mathrm{fr}$. 15 (1 drachme 1 obole). Celui des importateurs et revendeurs grecs n'était pas non plus à dédaigner, pucy le prix de la feuille variait à Délos entre $1 \mathrm{fr}$. 50 argent (1 drachme 3 oboles) et $2 \mathrm{fr}$. 15 (2 drachmes 1 obole).

On jugera par cette étude de détail combien il peut être utile de recueillir et de classer les chiffres, si rebutants d'apparence, qui hèrissent les comptes de nos inscriptions et de nos papyrus.

Il peut suffire de rappeler et de confronter une vingtaine de prix éparpillés sur deux siècles pour jeter un peu de lumière sur l'bistoire économique de I'antiquité, que les historiens du temps ont totalement négligée. C'est par une série d'études analogues qu'on aura quelques notions précises sur le commerce de détail et le commerce de gros, sur les conditions générales des échanges internationaux.

J'ai choisi comme exemple une denrée qui, par surcroit, renseigne sur l'histoire politique, voire même sur l'histoire intellectuelle des pays helléniques. Il n'est pas indifférent de constater un des effets produits subitement sur la situation matérielle de l'Égypte par la conquête macédonienne, une des différences profondes qui distinguèrent le règne de Ptolémée Sôter et celui de Ptolémée Philadelphe. Et l'on peut réfléchir longuement aux conséquences d'un système qui

1. Pap. Tebt., t. I, no 112, 1. 25 ; cf. 1. 61-62, 81-82.

2. Hermes, t. XX XVIII, 1913 , p. 403, n. 1 .

3. Op. cit., p. 67 . 
avait pour premier résultat de ne livrer à la consommation qu'un papier aussi cher que l'est dans les sociétés contemporaines le papier timbré. Le monopole avait à peine été supprimé par Alexandre, que l'Athénien Lycurgue, administrateur pourtant économe, fit faire et déposer aux archives une copie officielle des poètes tragiques, ce qui était un beau dèbut pour une Bibliothèque nationale. Mais le monopole rétabli par Philadelphe communiquait Ia cherté du papier au livre. Ainsi s'explique l'importance incomparable que prit immédiatement et que conserva pendant des siècles la Bibliothèque d'Alexandrie : les Ptolémées lui fournissaient le papyrus gratuitement ou à vil prix. En même temps, ils étaient les maitres de le vendre à l'étranger d'après un tarif fixé par eux seuls et même - c'est ce que fit Evergète II $^{1}$ - d'en interdire l'exportation.

Gustave Glotz.

1. Pline, XIII, 70.

(Paris.) 